\title{
THE MONITORING SYSTEM OF HAZARDOUS TECHNOLOGICAL OBJECTS BASED ON UNMANNED AERIAL VEHICLES
}

\author{
Ivan Zoev ${ }^{1 *}$, Nikolai Markov ${ }^{2}$, and Svetlana Ryzhova ${ }^{1}$ \\ ${ }^{1}$ National Research Tomsk Polytechnic University, Tomsk, Russia \\ ${ }^{2}$ Dr.Tech.Sci, National Research Tomsk Polytechnic University, Tomsk, Russia
}

\begin{abstract}
The paper describes the system for monitoring of hazardous technological objects, which based on unmanned aerial vehicles. The feature of the system is the using of the intelligent computer vision subsystem in which hardware-implemented a convolutional neural network based on a system-on-a-chip. This subsystem recognizes objects of the earth's surface and made decision about incidents at the monitoring area in real time. It also has service-oriented software architecture for groundbased system components. The paper presents results of the research of the required resources of a field-programmable gate array for implementation of error-correction encoder-decode based-on-board.
\end{abstract}

\section{Introduction}

Nowadays in Russia and many foreign industrialized countries have problems of monitoring the situations and detecting illegal actions at hazardous technological facilities. Also it has a problem of timely detection of fires at such facilities and in the territories they belong. Solve of these problems is primarily relevant for such industries, that have complex technological processes and facilities. The more popularity becomes unmanned aerial vehicles (UAVs) with special equipment for monitoring such facilities installed on them. Usually on a board UAVs are installed a video camera and thermal imager, which allows to shooting the monitoring territory. The existing monitoring systems don't analyze on the UAVs obtained images. These images are transmitted via radio-channel to the ground part of system and then operator analyzes (interprets) them. The lack of images real-time analysis on the UAV doesn't allow timely to make a decision in the ground part of monitoring system and send an alarming group to the place of the incident. As a result, all of this does not allow understanding the technical condition or prevent an invasion in a technological facility.

Earlier in our article [1] we proposed an idea of images analysis during the monitoring of earth's surface on a board UAVs in real-time (at the images receiving rate from a video camera and thermal imager). To realization of this idea we proposed to include an intelligent computer vision subsystem (ICVS) to the monitoring system and installed it on

*Corresponding author: zoev.ivan@yandex.ru 
the UAV. This subsystem will allow analyzing obtained data in automatic mode and made a decision about an incident on monitoring territory.

In this article we describe a concept of developed monitoring system of hazardous technological facilities based on modern UAVs and ICVS. This monitoring system may be autonomous or may be included in center of territories monitoring control. Also we will present features of its subsystems.

\section{Concept of monitoring system}

The system includes an UAV of aircraft-type or helicopter-type, ICVS installed on UAVs, ground-based information monitoring control subsystem and data transmission subsystem. Last of these subsystem allows transmit via radio-channel instructions to UAV and receive a monitoring data from its.

Choosing of UAVs type depends on technological facilities features to be monitored and the tasks, which will be solved using the system. UAV must include flight controller, GPS module, battery designed for significant flight time, engine(s) with speed controller and etc. Note that helicopter-type UAV can be located above the monitoring facilities and had power from the ground, because of its stationary location.

To monitor large areas, which includes several hazardous facilities (magistral oil and gas pipeline and etc.) may be developed a monitor control center. In this case information monitoring subsystem may be a part of this center and monitoring subsystem will not be autonomous. Operators of this control center should be able of quick interpretation of obtained from UAV images, if it necessary in doubt about an incident to made a final decision about sending of alarming group. Situation like this takes a place, because not all incidents may be identified by ICVS correctly. All these mean that the information subsystem should be integrated with one or several subsystems of automatic interpretation of obtained images in the center.

\section{Intelligent computer vision subsystem}

ICVS installed on UAV and included photo or video camera, thermal imager for detection of fire-sources and computing unite (CU). As a CU core we used a modern system on a chip (SoC). CU manages the collection of earth's surface objects images, analyze them using convolutional neural network $(\mathrm{CNN})$ and automatic decides about the presence of incidents in monitoring areas. The main requirement to ICVS is the performance of all these function in real-time. According the high requirements from $\mathrm{CNN}$ to $\mathrm{CU}$ performance, we proposed a hardware-based implementation of promising $\mathrm{CNN}$ architectures on Field Programmable Gate Array (FPGA) SoC.

We have implemented two variants of ICVS. First of these variants is hardwareimplemented CNN of original architecture from LeNet5 class on Cyclone V SX SoCs [2]. This CNN solving task of classification of objects of various physical natures (technological objects, special equipment, people and etc.) in images. The second variants of ICVS has CU with SoC Zynq 7000 (Kintex FPGA) as a core. On the FPGA of this Soc we hardware implemented an original tiny-YOLO-InceptionResNet2 CNN architecture of YOLO class [3].

We researched every of these hardware-implemented CNNs using dataset called "Stanford Drone Dataset" [4]. Results of these researchers helped to select of CNN hyperparameters in this way that to recognize objects in images with practically acceptable accuracy. Moreover each ICVS variants may function in real-time. 


\section{Information monitor control subsystem}

The information subsystem has a software (SW) of well-known freely geoinformation system (GIS) QGIS as a core [5]. Operators, who work with this subsystem, can plot and track UAVs routes, display the coordinates of incident (objects) in electronic map and etc using this SW. The information subsystem may be included in both an autonomous monitoring system and a monitoring control center with monitoring system.

We based on service-oriented architecture (SOA) principles when developed software for information subsystem and developed a unified information space of control center. Considering this, we developed SW center structure (fig.1). In figure 1 colored components, which belongs to information subsystem. The basis of SOA model are ELMA BMP business-processes control system and enterprise service bus ESB. They allow cooperating subsystem with third-party services, with database (through a database management system - DBSM) and with other systems automated interpretation systems of center, for example with ERDAS system. Figure 1 shows third-party web-services of errorcorrecting data encoding-decoding, included in ground-based part of data transmission subsystem. We have developed adapters for systems integration with ESB-bus.

We added an "Active UAV" module into standard SW QGIS, developed in its environment.

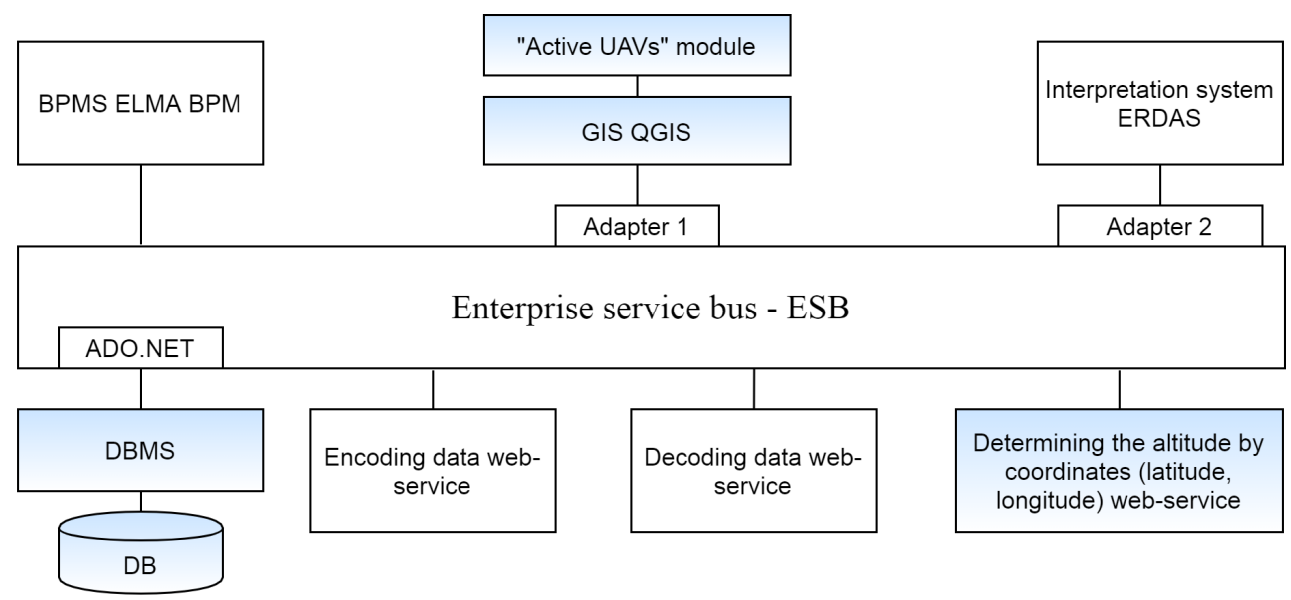

Fig. 1. Structure of SW of monitoring control center

The main module window is marked by rectangle in figure 2 . An ovals in this figure presents enlarged functions, which performed by module (the dotted line indicates, that function is running in background). And the hexagon shows QGIS core functions.

This module's functions of UAVs adding/removing, UAVs replacing and viewing of received images must connect with MS SQL Server 2017 DBMS, which allows maintaining the database (BD). This BD stores information about UAV, images transmitted from UAV to monitoring control center, and other service information. The function of tracking UAV in flight designed to route in real-time the UAV's flight on the map. This function has tight connection with QGIS core, which provides all necessary tools for displaying the route in the map. 


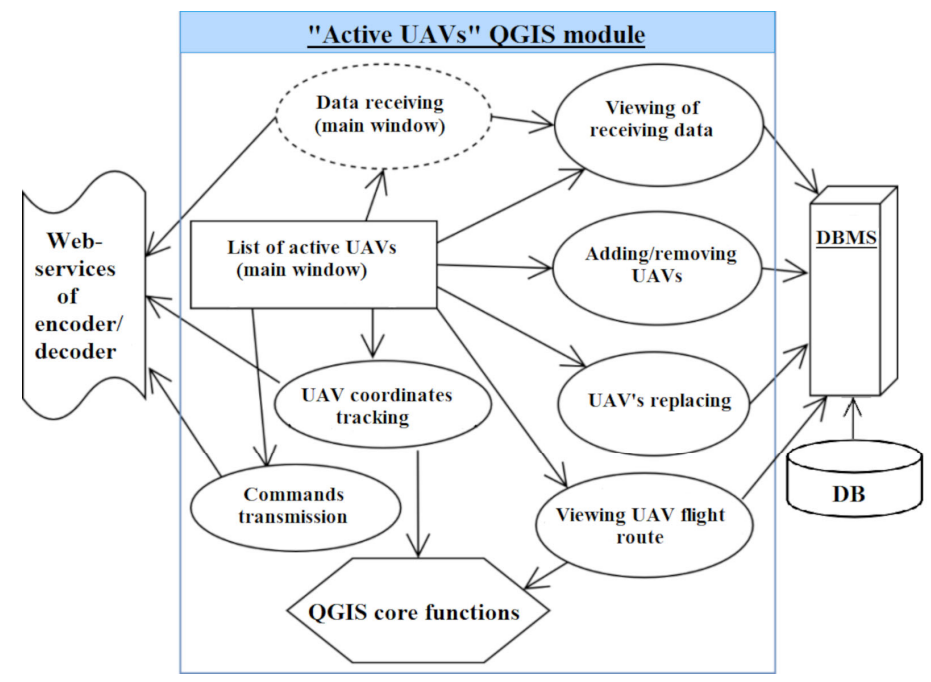

Fig. 2. The relationship diagram between module elements "Active UAVs"

Commands transmission to the UAV and data receiving from the UAV are carried out through web-services. These web-services carry out an error-correcting encoding and decoding of all transmitted and received information.

We have developed and have software implemented algorithms of cooperation of "Active UAVs" module with ICVS using C\# language. For developing an algorithm of transmission an image from UAV to information monitoring control subsystem we have took into account that the implementation of image viewing function requires the cooperation of the QGIS with DB. This cooperation was developed using SQL Python-pyodbc driver and QGIS PythonAPI $[5,6]$.

\section{Data transmission subsystem}

The exchange of commands and data between the onboard and ground-based parts of the data transmission subsystem is carried oud via a radio-channel. The onboard part in UAV has a radio-module and the ground-based part has a transceiver for such exchanges. To increasing of reliability of transmitted commands and data the onboard part and the groundbased part of data transmission subsystem can encode these data using error-correcting codes for detection and correction of errors in them. Among error-detection codes we have chosen a cyclic CRC code. Researches showed that the best option for CRC computation on FPGA is a matrix algorithm.

Encoder in the UAV make error-correction data encoding, which will be transmitted to the ground-based part of subsystem, using polynomial cyclic encoding algorithm. Figure 3 presents researches results of the Altera amounts FPGA resources requires by encoder depending from the data length $\mathrm{n}$, that counts in bits on the SoC Cyclone V SX.

We have researched lengths of data $n=31,63,127$ and 255 bits. The amount of necessary FPGA resources was determined by the number of required ALMs-blocks (adaptive logic modules) and ALUTs-blocks (adaptive look-up table). The analysis showed that for data length $n \leq 127$ bits the amounts of needed FPGA resources for encoder implementation is less than we can allocate for this encoder. Similar results of researches we have obtained for FPGA-based decoder, which using a cyclic decoding algorithm. 


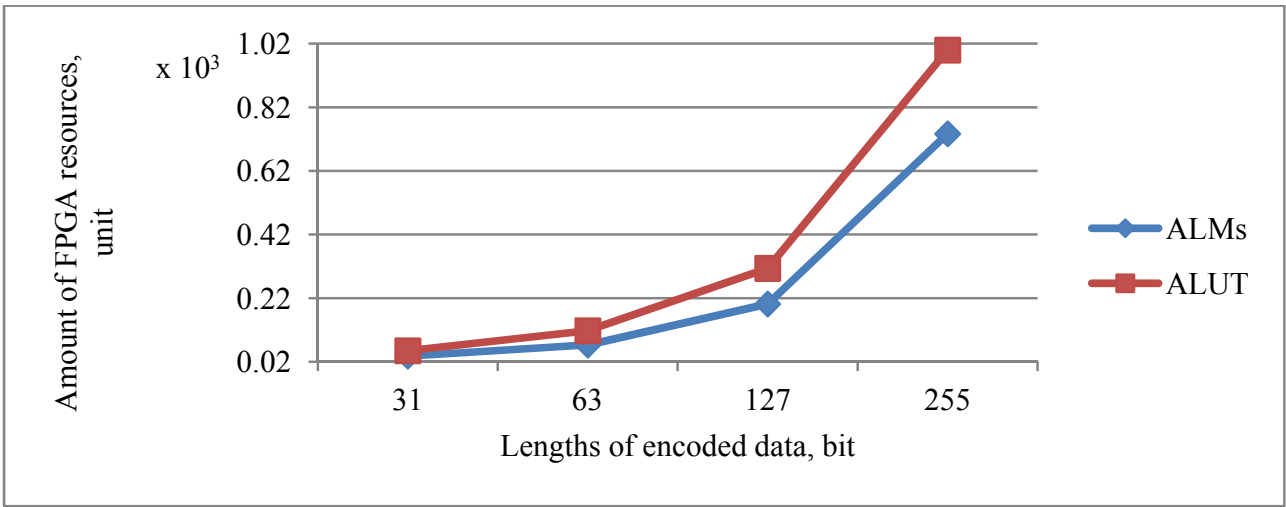

Fig. 3. Dependence of amounts of FPGA resources needed for ecnoder from length of encoding data

The ground-based part of data transmission subsystem has software-implemented of errorcorrecting encoder-decoder as web-services (fig.1) using C\# language.

We have researched the decoding speed of receiving form the UAV data depending on the encoded data lengths (n) and number of errors using table and cyclic decoding algorithms. Researches were carried out for 50 sending, each of them containing of $50 \mathrm{Kbit}$ of data, and for number of errors from 1 to 7 bits in a row. We may a conclusion based on this researches that decoding time using table algorithm increases insignificantly with an increasing of errors number in encoded data. But for cyclic algorithm in this case decoding time increases at 1.5 times. However, the table algorithm requires a significant memory amount for table storing. Moreover, this memory amount depends on data lengths and error correcting capability, and increases exponentially with the lengths. As a result, we have chosen a cyclic decoding algorithm for implementation. Table 1 shows results of researches decoding time using this algorithm. This table contains minimum, maximum and mean time, necessary for decoding of data various lengths (Tmin, Tmax and Tmean respectively).

Table 1. Result of researching of decoding data time using cyclic algorithm in ground-based part of transmission subsystem

\begin{tabular}{|l|c|c|c|}
\hline $\mathrm{n}$ & $\mathrm{T}_{\min }, \mathrm{sec}$ & $\mathrm{T}_{\max }, \mathrm{sec}$ & $\mathrm{T}_{\text {mean }}, \mathrm{sec}$ \\
\hline 31 & 9.088 & 9.126 & 9.101 \\
\hline 63 & 9.984 & 10.210 & 10.091 \\
\hline 127 & 20.754 & 20.895 & 20.812 \\
\hline 255 & 66.249 & 67.206 & 66.618 \\
\hline
\end{tabular}

These obtained results indicate that the optimal data lengths, received and decoded in ground-based data transmission subsystem part, is $n=127$ bits. For data with bigger length we have noticed a sharp increasing of time, needed for decoding, which doesn't fulfill the requirements of real-time monitoring system operation.

\section{Conclusion}

Nowadays the development of mobile and fundamentally new monitoring systems for hazardous technological facilities is important. We have developed the concept of such system based on UAV and have implemented its prototype. Also, we have tested this systems prototype in laboratory conditions and results of these test indicates about the efficiency of all its subsystems. 


\section{Acknowledgment}

The research was supported by Russian Foundation for Basis Research grant No. 18-47700010 r_a.

\section{References}

1. I.V. Zoev, N.G. Markov, S.E. Ryzhova Intelligent computer vision system for unmanned aerial vehicles for monitoring technological objects of oil and gas industry, Bulletin of Tomsk Polytechnic Uni. Geo Assets Eng, 330(11), (2019)

2. I.V. Zoev, A.P. Beresnev, N.G. Markov, A.N. Malchukov FPGA-based device for handwritten digit recognition in image, Computer Optics, 41(6) (2017)

3. I.V. Zoev, A.P. Beresnev, N.G. Markov Convolutional neural networks of the YOLO class in computer vision systems for mobile robotic complexes, Proc. IEEE Inter. Sib. Conf on Control and Comm.,

URL: https://ieeexplore.ieee.org/stamp/stamp.jsp?tp=\&arnumber=8729605

(2019)

4. Stanford Drone Dataset: Multi-scale, Multi-target social navigation, URL: https://stanford.resoluteinnovation.com/technologies/S17-376

5. Official site of QGIS, URL: https://www.qgis.org/ru/site/

6. QGIS 3 Plugins - Qt Designer for Plugins, URL: https://gis-ops.com/qgis-3-qtdesigner-explained 